\title{
Internalized-arginylglycylaspartic Acid Cyclic Peptide
}

National Cancer Institute

\section{Source}

National Cancer Institute. Internalized-arginylglycylaspartic Acid Cyclic Peptide. NCI

Thesaurus. Code C103858.

A 9 amino acid-based cyclic, tumor specific homing, arg inine-glycine-aspartic acid (RGD)based peptide (CRGDKRGPDC), with tumor penetrating activity. The IRGD contains the RGD motif as well as the C-terminal end binding (CendR) motif that increases internalization. Upon administration, the RGD motif of the IRGD peptide is able to specifically target tumors by binding to alphavbeta3/alphavbeta5 integrins on tumor endothelium. In turn, iRGD is cleaved by specific tumor proteases, which exposes the positively charged CendR motif. This motif binds to neuropilin-1 (NRP-1), a receptor overexpressed on certain tumor cells. This increases vascular permeability of tumor blood vessels and promotes tumor penetration. Compared to other RGD peptides, this agent is able to both improve delivery and increase the accumulation of co-administered or conjugated chemotherapeutic agents in the tumor. 